\title{
Outcomes of severe peripartum cardiomyopathy and mechanical circulatory support: a case series
}

\author{
Yuki Kiriyama', Yuki Kinishi', Daisuke Hiramatu', Akinori Uchiyama', Yuji Fujino', Koichi Toda² and \\ Chiyo Ootaki ${ }^{* *}$ (D)
}

\begin{abstract}
Background: We present three cases of severe peripartum cardiomyopathy (PPCM) that required mechanical circulatory supports.

Case presentation: Case 1: A 33-year-old woman developed acute heart failure (AHF) after normal spontaneous delivery. Intra-aortic balloon pump (IABP) was inserted on postpartum day (PD) 10 with a peripartum cardiomyopathy (PPCM), which was withdrawn on PD 30 after medical treatment including anti-prolactin drugs.

Case 2: A 44-year-old woman developed AHF 1 month after vaginal delivery. IABP or extra-corporeal membrane oxygenation (ECMO) was not effective and a biventricular assist device was inserted. It was withdrawn on PD 85 after improvement of left ventricular ejection fraction (LVEF).

Case3: A 37-year-old woman was transferred with a diagnosis of PPCM. Cardiac function unimproved by IABP or ECMO, and a left ventricular assist device was implanted. It was withdrawn on PD 386 after recovery of LVEF.

Conclusion: All the cases with PPCM recovered after mechanical circulatory supports and resumed social lives.

Keywords: Cardiomyopathy, Heart failure, Pregnancy, Mechanical circulatory support, Bridge to transplantation, Bridge to recovery
\end{abstract}

\section{Background}

Peripartum cardiomyopathy (PPCM) is a heart failure secondary to reduced contractility due to unknown etiology during the peripartum period in previously healthy women. It is relatively rare with an incidence of one case per 10,000-20,000 deliveries [1]. Patients are generally treated for heart failure, and approximately half of them recover $[2,3]$. However, patients who maintain cardiomegaly for 6 months or longer have an extremely poor prognosis [2].

*Correspondence: ootaki@anes.med.osaka-u.ac.jp

${ }^{1}$ Department of Anesthesiology and Intensive Care, Osaka University Graduate School of Medicine, 2-2 Yamadaoka, Suita City, Osaka 565-0871, Japan

Full list of author information is available at the end of the article
There are several types of mechanical circulatory supports (MCSs) and are typically classified as either temporary or durable [4]. Temporary MCS devices include intra-aortic balloon pump (IABP), veno-arterial extracorporeal membrane oxygenation (VA-ECMO), and temporary ventricular assist devices (VAD). Durable MCS devices include implantable VADs that are used as destination therapy for end-stage cardiac failure. There are a few reports on the use of MCS for PPCM, and no studies on it appreciate induction timing of MCS or outcomes [5, 6]. Herein, we present three cases of severe PPCM that required MCS.

\section{Case presentation}

Over the past 5 years, we encountered three patients with severe PPCM who were unresponsive to medical therapy and required MCS. The profile of the three cases 
who required MCSs are presented in Table 1, treatment details in Table 2, and cardiac data in Table 3.

\section{Case 1}

A 33-year-old woman (G1P1; height, $156 \mathrm{~cm}$; weight, $46 \mathrm{~kg}$ ) without a significant medical history developed malaise on postpartum day 7 after normal spontaneous delivery. Her symptom was dyspnea and felling chill when she was referred to cardiology on postpartum day 10. At the time of the cardiology, BNP $930 \mathrm{pg} / \mathrm{ml}$, echocardiography showed LVEF $24 \%$, LVDd/Ds $63 / 58 \mathrm{~mm}$, moderate mitral regurgitation, blood samples showed mildly elevated liver enzymes and metabolic acidosis. Acute heart failure associated with PPCM was suspected. Myocardial biopsy and coronary angiography were performed, and coronary artery stenosis was excluded. Inotropes, diuretics, and an IABP were inserted on the same day (INTERMAX profile 2). Her hemodynamics was stabilized, but his heart failure worsened with fever, and he was transported to our hospital for LVAD induction on postpartum day 16. At our hospital, with the use of continuous hemodiafiltration, inotropes, and anti-prolactin drugs, the cardiac function LVEF recovered to $30 \%$, CO $2.3 \mathrm{~L} / \mathrm{min}$. IABP was withdrawn postpartum day 30 . The patient was discharged from the intensive care unit with medical treatment.

\section{Case 2}

A 44-year-old woman (G5P5; height, $151 \mathrm{~cm}$; weight, $56 \mathrm{~kg}$ ) with left bundle branch block developed acute heart failure and acute pulmonary edema postpartum day 25 after normal vaginal delivery of her fifth child. She was diagnosed PPCM and medical treatment was started with catecholamines, diuretics, and anti-prolactin drugs and fluid restriction. The patient was hemodynamically stabilized with diuretics and inotropes. Postpartum day 43 , her symptoms worsened due to noninfectious fever and developed cardiac shock with LVEF of $22 \%$ and LVEDd of $67 \mathrm{~mm}$ and CO $2.16 \mathrm{~L} / \mathrm{min}$. Postpartum day 44, IABP was induced, but the shock status unchanged, PCPS was introduced and transported to our hospital. IABP and VA-ECMO were started. Based on the indication for cardiac transplantation (INTERMAX profile 1) and marked pulmonary edema, the extracorporeal biventricular assist device was inserted postpartum day 45. Subsequently, LVEF improved to $40 \%$ and $\mathrm{CO} 3.47 \mathrm{~L} / \mathrm{min}$; therefore, the right ventricular assist device was withdrawn postpartum day 71 and cardiac resynchronization therapy defibrillator (CRT-D) with biventricular pacing was initiated for residual heart failure. Postpartum day 85 , the left ventricular assist device (LVAD) was withdrawn, and the patient was discharged from the intensive care unit.

\section{Case 3}

A 37-year-old woman (G1P1; height, $158 \mathrm{~cm}$; weight, $78 \mathrm{~kg}$ ) without a significant medical history was referred to our hospital with a diagnosis of PPCM. Postpartum day 4, she developed dyspnea with lower leg edema, shock, and LVEF was $33 \%$ (INTERMAX profile 1). Medical treatment was started with inotropes and diuretics for heart failure. Subsequently, LVEF reduced to $20 \%$, LVEDd was $69 \mathrm{~mm}$, and mitral regurgitation worsened. IABP, VA-ECMO, and anti-prolactin therapy were started on postpartum day 7. However, congestion progressed with dilation of the left ventricle and left ventricular vent was inserted on postpartum day 11 , which resulted in improvement of congestion. Postpartum day 19, VA-ECMO was stopped due to thrombus in its circulation, and at this time the LVEF was $35 \%$ and CO $4.76 \mathrm{~L} / \mathrm{min}$. Postpartum day 23, we had no choice but to removed IABP and continued medical treatment. However, the cardiac dysfunction could not be controlled and the patient needed IABP again, postpartum day 118. Postpartum day 129 , the implantable LVAD was implanted and mitral valve replacement was performed. Postpartum day 386 , LVEF recovered to $55 \%$ and $\mathrm{CO}$ $4.36 \mathrm{~L} / \mathrm{min}$, and the LVAD was withdrawn and the patient was discharged from the hospital.

Table 1 Profiles of 3 cases

\begin{tabular}{llll}
\hline & Case 1 & Case 2 & Case 3 \\
\hline Age of PPCM onset, years & 33 & 44 & 37 \\
BMl, kg/m & 18.9 & 24.5 & 28.4 \\
Pregnancy history & Gravida 1, Para 1 & Gravida 5, Para 5 & Gravida 1, Para 1 \\
Family history & None & None & Mother: DCM \\
Delivery & Vaginal & Vaginal & Vaginal \\
& Single-fetal & Single-fetal & Single-fetal
\end{tabular}

PPCM peripartum cardiomyopathy, $B M I$ body mass index, $D C M$ dilated cardiomyopathy 
Table 2 Treatment details of 3 cases

\begin{tabular}{llll}
\hline & Case 1 & Case 2 & Case 3 \\
\hline Onset (postpartum) & PD 7 & PD 25 & PD 4 \\
Bromocriptine & O & O & O \\
MCS & IABP & IABPs & IABP \\
& & VA-ECMO & LV -EMO \\
& & Temporary-BiVAD & LVAD \\
IABP, VA-ECMO implantation after onset & 3day (PD 10) & 19 days (PD 44) & 3days (PD 7) \\
VAD implantation after onset & - & 20 days (PD 45) & 125 days (PD 129) \\
MCS outcome & Withdrawal & Withdrawal & Withdrawal \\
MCS implant duration (MCS withdrawal day) & 20 days (PD 30) & 41 days (PD 85) & 286 days (PD 386) \\
Outcome & Survival & Survival & Survival \\
\hline
\end{tabular}

MCS mechanical circulatory support, IABP intra-aortic balloon pump, VA-ECMO veno-arterial extracorporeal membrane oxygenation, BiVAD biventricular assist device, VA-ECMO veno-arterial extracorporeal membrane oxygenation, $L V A D$ left ventricular assist device, $P D$ postpartum day

\section{Discussion}

The diagnosis of PPCM is based on the following Demakis criteria: new symptoms of heart failure 1 month before and within 5 months after delivery; no history of heart disease; no other causes of heart failure; and positive echocardiographic findings (LVEF $<45 \%$, fractional shortening of left ventricular diameter, and $\mathrm{FS}<30 \%$ ) [2]. The cause of PPCM remains unknown; however, there are several theories, such as genetic predisposition, viral infections, and inhibition of angiogenesis and cardiotoxicity by truncated prolactin [2]. The risk factors of PPCM include multiple births, age $>30$ years, eclampsia, obesity, and complicated gestational hypertension [1-3]. All patients in this report were over 30 years of age. PPCM is relatively rare in Japan but accounts for $3.9 \%$ of all maternal deaths $[1,7]$. Fortunately, cardiac function is expected to improve in PPCM compared to that in ischemic heart disease, and approximately half of patients with PPCM are expected to recover within 6 months [2]. Poor prognostic factors for PPCM include LVEF $<30 \%$ and LVEDd $>60 \mathrm{~mm}$; they indicate a reduced rate of improvement in left ventricular function and an increased risk of invasive treatments, transplantation, and death [8]. All three patients who required MCS in this study had poor prognostic factors.

The first step in the treatment of PPCM is to treat it as general heart failure and arrhythmia. Additionally, prolactin inhibitor has been demonstrated to be effective. Prolactin inhibitor therapy was administered in all three cases in this study. Additionally, PPCM requires concomitant anticoagulation therapy due to the high incidence of thrombosis [9].

Although there are no clear criteria for inducing MCS for PPCM, NYHA III or IV, increased diuretic requirements, dependence on circulatory agonists, organ failure

Table 3 Pre- and post-MCS cardiac function

\begin{tabular}{|c|c|c|c|}
\hline & Case 1 & Case 2 & Case 3 \\
\hline MCS & IABP & $\begin{array}{l}\text { IABPs } \\
\text { VA-ECMO } \\
\text { Temporary-BiVAD }\end{array}$ & $\begin{array}{l}\text { IABP } \\
\text { VA-ECMO } \\
\text { LVvent } \\
\text { LVAD }\end{array}$ \\
\hline INTERMACS before MCS and symptoms & Profile 2 & Profile 1 & Profile1 \\
\hline Pre-MCS LVEDd (mm) & 63.2 & 67 & 69 \\
\hline Pre-MCS LVEF (\%) & 19.4 & 22 & 22 \\
\hline Pre-MCS CO (L/min) & - & 3.26 & 3.47 on PCPS, IABP \\
\hline Pre-MCS PAP (s/d/m: mmHg) & $16 / 10(13)$ & $49 / 20(27)$ & 43/24 (32) on PCPS, IABP \\
\hline Pre-MCS LVEDd (mm) & 65 & 46 & \\
\hline Post-MCS LVEF (\%) & 23 & 32 & 56 \\
\hline Post-MCS CO (L/min) & 3.9 & 4.52 & 4.36 \\
\hline Pre-MCS PAP (s/d/m: mmHg) & $21 / 6(13)$ & 21/11 (15) & $35 / 15(24)$ \\
\hline
\end{tabular}

LVEF left ventricular ejection fraction, LVEDd left ventricular end-diastolic diameter, $C O$ cardiac output, PAP pulmonary atrial pressure, MCS mechanical circulatory support, $P C P S$ percutaneous cardiopulmonary support, IABP intra-aortic balloon pump, VA-ECMO veno-arterial extracorporeal membrane oxygenation, BiVAD biventricular assist device, LVAD left ventricular assist device 
Table 4 INTERMACS clinical profile [4]

\begin{tabular}{|c|c|c|}
\hline Level & Description & Hemodynamic status \\
\hline 1 & Critical cardiogenic shock, "crash and burn" & $\begin{array}{l}\text { Persistent hypotension despite rapidly escalating inotropic support and even- } \\
\text { tually IABP, and critical organ hypoperfusion }\end{array}$ \\
\hline 2 & Progressive decline on inotropic support, "sliding on inotropes" & $\begin{array}{l}\text { Intravenous inotropic support with acceptable values of blood pressure and } \\
\text { continuing deterioration in nutrition, renal function, or fluid retention }\end{array}$ \\
\hline 3 & Stable but inotrope dependent, "dependent stability" & $\begin{array}{l}\text { Stability reached with mild to moderate doses of inotropes but demonstrating } \\
\text { failure to wean from them because of hypotension, worsening symptoms, or } \\
\text { progressive renal dysfunction }\end{array}$ \\
\hline 4 & Resting symptoms, "frequent flyer" & $\begin{array}{l}\text { Possible weaning of inotropes but experiencing recurrent relapses, usually fluid } \\
\text { retention }\end{array}$ \\
\hline 5 & Exertion intolerant, housebound & $\begin{array}{l}\text { Severe limited tolerance for activity, comfortable at rest with some volume } \\
\text { overload and often with some renal dysfunction }\end{array}$ \\
\hline 6 & Exertion limited, "walking wounded" & $\begin{array}{l}\text { Less severe limited tolerance for activity and lack of volume overload, fatigue } \\
\text { easily }\end{array}$ \\
\hline 7 & Advanced NYHA III "symptoms, placeholder" & Patient without current or recent unstable fluid balance, NYHA class II or III \\
\hline
\end{tabular}

INTERMACS Interagency Registry for Mechanically Assisted Circulatory Support, NYHA New York Heart Association functional classification

due to poor perfusion, and LVEF $<30 \%$ are considered to use MCS to stabilize their general condition [4]. INTERMACS is a US registry acquiring data on patients supported with FDA-approved MCS devices. Within INTERMACS, patients are classified by their signs and symptoms into 7 clinical profiles (1 is most severe and 7 is less severe, Table 4), and the INTERMACS profiles provide guidance for the optimal timing of MCS implantation [10]. Basically, profile 1 cases are indicated for extracorporeal LVADs, and in profile 2, 3 cases are indicated for implantable LVADs [10]. Temporary circulatory procedures such as IABP, ECMO, and Impella are indicated in INTERMACS profiles 1, 2, and 3 [10]. Profiles 6 or 7 patients are, in general, considered too well for MCS.

In this reports, one patient was INTERMACS profile 2 and other were 1 at the time of MCS induction, and the profile 2 case induced IABP at the same day of cardiology visit before heart failure progress.

Traditionally, durable MCSs were reserved primarily for patients with end-stage heart failure who were deemed candidates for transplantation as "bridge to transplantation" and long-term destination therapy for non-transplant candidates in Japan and other countries with a shortage of transplant donors [11, 12]. In contrast, VAD may be implanted for recovery of own heart function; subsequent withdrawal of VAD is called "bridge to recovery (BTR)" [13-17]. By reducing the hemodynamic pre-load and resting the myocardium using an implanted VAD, the original cardiac function is restored and the VAD can be withdrawn. There are a few case reports of successful BTR using the LVAD in PPCM $[5,6]$. Especially regarding to non-ischemic cardiomyopathy, a characteristic of patients with successful BTR is the early introduction to VAD support [18]. In the BTR report on non-ischemic myocardium,
73\% of patients who had an LVAD implanted within the first 4 months of heart failure symptoms recovered, while no recovery was seen in subjects who had more than 4 months [18]. In this report, two cases requiring LVADs. In our case 3, IABP was introduced early, but it took a long time to introduce LVAD. The patient eventually recovered, but it took longer time for treatment than case 2 which induced LAVD within 4 months of onset, at 18 days after onset. Our observations suggest the possibility of improving recovery and prognosis in PPCM using early MCSs. Although the invasive nature of the LVAD surgery may raise the hurdle, the introduction of MCS for PPCM that does not respond to medical therapy should be done aggressively. PPCM that does not respond to medical therapy should be referred to a center with expertise in the management of MCS and patients with advanced PPCM heart failure.

\section{Conclusion}

We presented three cases of severe PPCM that required MCS. All the patients survived and resumed their social lives.

\section{Abbreviations}

PPCM: Peripartum cardiomyopathy; MCS: Mechanical circulatory support; IABP: Intra-aortic balloon pump; PCPS: Percutaneous caidiopulmonary support; VA-ECMO: Veno-arterial extra-corporeal membrane oxygenation; BiVAD: Biventricular assist device; LVEF: Left ventricular ejection fraction; LVAD: Left ventricular assist device; VAD: Ventricular assist devices; LVEDd: Left ventricular end-diastolic diameter; CRT-D: Cardiac resynchronization therapy defibrillator; BTR: Bridge to recover; NYHA: New York Heart Association functional classification.

\section{Acknowledgements}

The authors would like to extend their gratitude to the patients, their family members, and the obstetricians and cardiologists of Osaka University. 


\section{Authors' contributions}

YK drafted the manuscript. YKs, DH, and AU managed for those cases. UF is director of intensive care units. KT is operator of MCS. CO supervised the drafting. All authors read and approved the final version of the manuscript.

\section{Funding}

The authors received no external funding.

\section{Availability of data and materials}

Please contact the corresponding author for data requests.

\section{Declarations}

Ethics approval and consent to participate

Not applicable.

\section{Consent for publication}

Informed consent for scientific publication was obtained from the patient.

\section{Competing interests}

The authors declare that they have no competing interests.

\section{Author details}

${ }_{1}^{1}$ Department of Anesthesiology and Intensive Care, Osaka University Graduate School of Medicine, 2-2 Yamadaoka, Suita City, Osaka 565-0871, Japan.

${ }^{2}$ Department of Cardiovascular Surgery, Osaka University Graduate School of Medicine, Osaka, Japan.

Received: 30 July 2021 Revised: 12 October 2021 Accepted: 13 October 2021

Published online: 02 November 2021

\section{References}

1. Kamiya CA, Kitakaze M, Ishibashi-Ueda H, et al. Different characteristics of peripartum cardiomyopathy between patients complicated with and without hypertensive disorders. Results from the Japanese Nationwide survey of peripartum cardiomyopathy. Circ J. 2011;75:1975-81.

2. Demakis JG, Rahimtoola SH. Peripartum cardiomyopathy. Circulation. 1971;44:964-8.

3. Sliwa K, Fett J, Elkayam U. Peripartum cardiomyopathy. Lancet. 2006;368:687-93.

4. Peura JL, Colvin-Adams M, Francis GS, et al. Recommendations for the use of mechanical circulatory support: device strategies and patient selection: a scientific statement from the American Heart Association. Circulation. 2012;126:2648-67.
5. Oosterom L, de Jonge N, Kirkels JH, Klöpping C, Lahpor JR. Left ventricular assist device as a bridge to recovery in a young woman admitted with peripartum cardiomyopathy. Neth Heart J. 2008;16:426-8.

6. Lund LH, Grinnemo KH, Svenarud P, van der Linden J, Eriksson MJ. Myocardial recovery in peri-partum cardiomyopathy after continuous flow left ventricular assist device. J Cardiothorac Surg. 2011;6:150.

7. Isogai T, Kamiya CA. Worldwide incidence of peripartum cardiomyopathy and overall maternal mortality. Int Heart J. 2019;60:503-11.

8. Honigberg MC, Givertz MM. Peripartum cardiomyopathy. BMJ. 2019;30(364):k5287.

9. Zolt A, Uri E. Peripartum cardiomyopathy. Circulation. 2016;133:1397-409.

10. Stevenson LW, Pagani FD, Young JB, et al. INTERMACS profiles of advanced heart failure: the current picture. J Heart Lung Transplant. 2009:28:535-41.

11. MCCarthy PM, Smedira NO, Vargo RL, et al. One hundred patients with the HeartMate left ventricular assist device: evolving concepts and technology. J Thorac Cardiovasc Surg. 1998;115:904-12.

12. Rose EA, Gelijns AC, Moskowitz AJ, et al. Randomized evaluation of mechanical assistance for the treatment of congestive heart failure (REMATCH) study group. Long-term mechanical left ventricular assistance for end-stage heart failure. N Engl J Med. 2001;345:1435-43.

13. Gorcsan J 3rd, Severyn D, Murali S, Kormos RL. Non-invasive assessment of myocardial recovery on chronic left ventricular assist device: results associated with successful device removal. J Heart Lung Transplant. 2003:22:1304-13.

14. Farrar DJ, Holman WR, McBride LR, et al. Long-term follow-up of Thoratec ventricular assist device bridge-to-recovery patients successfully removed from support after recovery of ventricular function. J Heart Lung Transplant. 2002;21:516-21.

15. Khan T, Delgado RM, Radovancevic B, et al. Dobutamine stress echocardiography predicts myocardial improvement in patients supported by left ventricular assist devices (LVADs): hemodynamic and histologic evidence of improvement before LVAD explantation. J Heart Lung Transplant. 2003;22:137-46.

16. Frazier $\mathrm{OH}$, Reynolds $\mathrm{M}$, Scroggins $\mathrm{N}$, et al. Mechanical bridging to improvement in severe acute "nonischemic, nonmyocarditis" heart failure. Congest Heart Fail. 2004;10:109-13.

17. Simon MA, Kormos RL, Murali S, et al. Myocardial recovery using ventricular assist devices: prevalence, clinical characteristics, and outcomes. Circulation. 2005;112(9 Suppl):132-6.

18. Boehmer JP, Starling RC, Cooper LT, et al. IMAC investigators. Left ventricular assist device support and myocardial recovery in recent onset cardiomyopathy. J Card Fail. 2012;18:755-61.

\section{Publisher's Note}

Springer Nature remains neutral with regard to jurisdictional claims in published maps and institutional affiliations. 